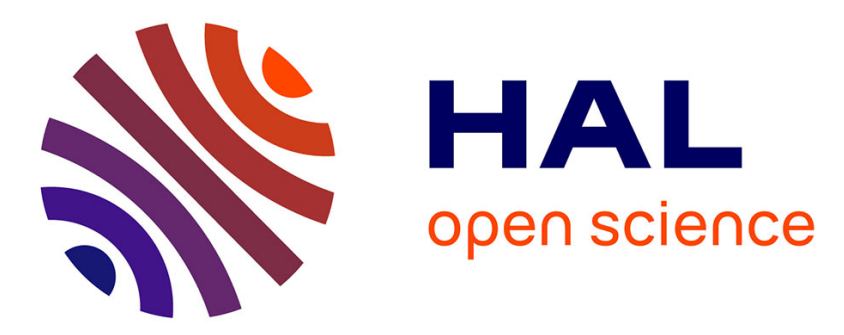

\title{
BOOTSTRAP-BASED BIAS REDUCTION FOR THE ESTIMATION OF THE SELF-SIMILARITY EXPONENTS OF MULTIVARIATE TIME SERIES
}

H. Wendt, Patrice Abry, G. Didier

\section{- To cite this version:}

H. Wendt, Patrice Abry, G. Didier. BOOTSTRAP-BASED BIAS REDUCTION FOR THE ESTIMATION OF THE SELF-SIMILARITY EXPONENTS OF MULTIVARIATE TIME SERIES. IEEE International Conference on Acoustics, Speech and Signal Processing (ICASSP), May 2019, Brighton, United Kingdom. hal-02346739

\section{HAL Id: hal-02346739 \\ https://hal.science/hal-02346739}

Submitted on 7 Nov 2019

HAL is a multi-disciplinary open access archive for the deposit and dissemination of scientific research documents, whether they are published or not. The documents may come from teaching and research institutions in France or abroad, or from public or private research centers.
L'archive ouverte pluridisciplinaire HAL, est destinée au dépôt et à la diffusion de documents scientifiques de niveau recherche, publiés ou non, émanant des établissements d'enseignement et de recherche français ou étrangers, des laboratoires publics ou privés. 


\title{
BOOTSTRAP-BASED BIAS REDUCTION FOR THE ESTIMATION OF THE SELF-SIMILARITY EXPONENTS OF MULTIVARIATE TIME SERIES
}

\author{
H. Wendt ${ }^{1}$, P. Abry ${ }^{2}$, G. Didier ${ }^{3}$ \\ ${ }^{1}$ IRIT, CNRS (UMR 5505), Université de Toulouse, France. \\ ${ }^{2}$ Univ Lyon, ENS de Lyon, Univ Claude Bernard, CNRS, Laboratoire de Physique, Lyon, France. \\ ${ }^{3}$ Math. Dept., Tulane University, New Orleans, USA.
}

\begin{abstract}
Self-similarity has become a well-established modeling framework in several fields of application and its multivariate formulation is of ever-increasing importance in the Big Data era. Multivariate Hurst exponent estimation has thus received a great deal of attention recently, in particular by means of the wavelet eigenvalue regression method. The present work tackles the issue of the presence of significant finite-sample bias in wavelet eigenvalue regression stemming from the eigenvalue repulsion effect, whose origin and impact are analyzed and quantified. Furthermore, an original wavelet domain bias reduction technique is developed assuming a single multivariate time series is available. The protocol consists of a bootstrap resampling scheme that preserves the joint covariance structure of multivariate wavelet coefficients. Extensive numerical simulations show that this proposed method is effective in counteracting the bias at the price of a small increase in variance. This leads to wavelet eigenanalysisbased estimation of multivariate Hurst exponents with significantly improved finite-sample performance than earlier state-of-the-art formulations.
\end{abstract}

Index Terms - multivariate self-similarity, wavelet spectrum, eigenvalue distribution, bootstrap

\section{INTRODUCTION}

Self-similarity. Over the last many decades, self-similarity has become a canonical paradigm in the analysis and modeling of dynamic behavior in a wide range of applications [1]. The most popular self-similarity model is fractional Brownian motion (fBm), namely, the only Gaussian, self-similar, stationary-increment stochastic process $[2,3]$. In the fBm framework, dynamics are parsimoniously characterized by means of a single parameter, the so-named Hurst exponent $H \in(0,1)$. In applications, this parameter can be used in classification, detection, diagnosis, and various other classical signal processing tasks. This generates a strong demand for accurate and robust statistical estimation of $H$ and, accordingly, a large body of work on the subject has been developed $[4,5]$. It is now well established that wavelet analysis can be used in the construction of estimation protocols for $H$ that are accurate, efficient and robust both in theory and in practice [6,7]. Nevertheless, until recently, the focus of the statistical literature was on the modeling and analysis of single, or univariate, time series. This explains why success in modeling has mostly remained restricted to univariate data settings. By contrast, in an ever-increasing number of areas, data are recorded by multiple sensors that jointly monitor physical systems over time. This has

Work supported by French ANR BLANC MULTIFRACS. G.D. was partially supported by the ARO grant W911NF-14-1-0475. lead to the availability of large and complex data sets, which, in turn, call for new statistical protocols that are multivariate from inception.

Self-similarity in multivariate data. Operator fractional Brownian motion (ofBm) was recently proposed as a framework for multivariate self-similarity [8-11]. For modeling purposes, ofBm can be viewed as a mixture of $M$ correlated $\mathrm{fBm}$ processes, each with possibly distinct self-similarity exponents $H_{m}, m=1, \ldots, M$. OfBm has now been successfully applied in many fields such as in Internet traffic modeling [12] and dendrochronology [13].

For ofBm, univariate-like statistical methods such as the entrywise analysis of sample covariance matrices, Fourier or wavelet spectra does not yield relevant results. In $[12,14]$, a wavelet eigenvalue regression methodology is constructed for the joint estimation of the vector of ordered Hurst exponents $\underline{H}=\left(H_{1}, \ldots, H_{M}\right)$. The protocol relies on the striking fact of the joint eigenvalue scaling property. This property states that, as $N \gg 2^{j} \rightarrow \infty$, the random eigenvalues $\lambda_{m}$ of sample wavelet covariance matrices $S\left(2^{j}\right)$ jointly scale according to power laws driven by their respective Hurst exponents, i.e., $\vartheta_{m}\left(S\left(2^{j}\right)\right) \stackrel{P}{\sim} C_{m} 2^{j\left(2 H_{m}+1\right)}, m=1, \ldots, M$.

However, over finite sample sizes $N$ and scales $2^{j}$, bias is always present as a function of the underlying ofBm parameters. In addition, wavelet eigenvalues driven by equal Hurst exponents tend to differ only by a constant factor. Hence, they are particularly susceptible to the so-named eigenvalue repulsion effect. As described in the random matrix literature $[15,16]$, eigenvalues of random Hermitian matrices tend to behave as if there was some force preventing them from getting too close to each other. For $S\left(2^{j}\right)$, this effect becomes more pronounced over large scales, for which the ratio between the dimension $M$ and the number of sum terms $N / 2^{j}$ entering the average $S\left(2^{j}\right)$ is large (cf. [17]). Yet, scaling analysis requires the use of large scales $2^{j}$ as to reveal the underlying low frequency dynamics.

Goal, contributions and outline. In this work, we tackle the issue of the presence of bias in finite-sample wavelet eigenvalue regression. We develop and study a bias reduction procedure assuming a single finite-length multivariate time series is available for the ofBm model and wavelet eigenvalue regression method, which are defined in Section 2. At the core of the procedure is an original bootstrap resampling scheme in the wavelet domain that preserves the joint covariance structure of multivariate wavelet coefficients, described in Section 3. Comprehensive numerical simulation results demonstrate that the proposed method is operational and effective in counteracting the bias originated in eigenvalue mutual repulsion, leading to significantly improved finite-sample estimation performance, cf. Section 4 . We provide conclusions and future work directions in Section 5. 


\section{MULTIVARIATE SELF-SIMILARITY}

\subsection{Model: operator fractional Brownian motion}

In this work, we make use of a subclass of time reversible ofBms that is sufficiently flexible for most practical situations (see [10] for a general definition of ofBm). Let $X \triangleq\left\{X_{H_{1}}(t), \ldots, X_{H_{M}}(t)\right\}_{t \in \mathbb{R}}$ be a collection of $M$ possibly correlated fBms with self-similarity exponents $\underline{H}=\left(H_{1}, \ldots, H_{M}\right), 0<H_{1} \leq \ldots \leq H_{M}<1$. Also, let $W$ be a real-valued, invertible $M \times M$ matrix. The $M$-variate ofBm is defined as the stochastic process

$$
\begin{gathered}
Y \triangleq\left\{Y_{1}^{\underline{H}, \Sigma_{X}, W}(t), \ldots, Y_{M}^{\frac{H}{M}, \Sigma_{X}, W}(t)\right\}_{t \in \mathbb{R}} \triangleq \\
W\left\{X_{H_{1}}(t), \ldots, X_{H_{M}}(t)\right\}_{t \in \mathbb{R}}=W X .
\end{gathered}
$$

In (1), $\Sigma_{X}=\mathbb{E} X(1) X(1)^{*}$ denotes the pointwise covariance matrix of $X$. Its entries can be reexpressed as $\left(\Sigma_{X}\right)_{m, m^{\prime}}=\sigma_{m} \sigma_{m^{\prime}} \rho_{m, m^{\prime}}$, where $\sigma_{m}^{2}$ and $\rho_{m, m^{\prime}}$ are the variances of each component and their respective correlation coefficients. $\underline{H}$ and $\Sigma_{X}$ can in general not be chosen independently [10]. Definition (1) implies that $Y$ has stationary increments and satisfies the multivariate self-similarity relation

$$
\begin{aligned}
\forall a>0 & : \quad\left\{Y_{1}^{\underline{H}, \Sigma_{X}, W}(t), \ldots, Y_{M}^{\underline{H}, \Sigma_{X}, W}(t)\right\}_{t \in \mathbb{R}} \\
& \stackrel{\text { fdd }}{\simeq}\left\{a \underline{\underline{H}}\left(Y_{1}^{\underline{H}, \Sigma_{X}, W}(t / a), \ldots, Y_{M}^{\underline{H}, \Sigma_{X}, W}(t / a)\right)\right\}_{t \in \mathbb{R} .}
\end{aligned}
$$

In (2), $\stackrel{\text { fdd }}{\approx}$ stands for the convergence of finite dimensional distributions, and $\underline{H} \triangleq W \operatorname{diag}(\underline{H}) W^{-1}$ is the so-called Hurst matrix, where $a \underline{\underline{H}} \triangleq \overline{\bar{\sum}}_{k=0}^{+\infty} \log ^{k}(a) \underline{\underline{H}}^{k} / k !$.

\subsection{Wavelet based joint estimation of $H_{m}, m=1, \ldots, M$}

Given a $M$-variate time series $Y$, the goal of self-similarity analysis is to infer the vector of exponents $\underline{H}=\left(H_{1}, \ldots, H_{M}\right)$ from the data. If it can be assumed that each component $Y_{m}$ is a $\mathrm{fBm}$ (i.e., the mixing matrix $W$ and, consequently, the Hurst matrix $\underline{\underline{H}}$ are diagonal), it is possible to estimate $\underline{H}$ using conventional, univariate-like approaches $[18,19]$. However, in the general case when components are mixtures of fBms, i.e., when $W$ is non-diagonal, univariatelike estimation of $\underline{H}$ is, in general, strongly biased. In [12, 14], a wavelet-based multivariate statistical strategy is proposed. The multivariate discrete wavelet transform coefficients are computed as $\left(D\left(2^{j}, k\right)\right) \triangleq D_{Y}\left(2^{j}, k\right)=\left(D_{Y_{1}}\left(2^{j}, k\right), \ldots, D_{Y_{M}}\left(2^{j}, k\right)\right), \forall k \in$ $\mathbb{Z}, \forall j \in\left\{j_{1}, \ldots, j_{2}\right\}$, with $\forall m \in\{1, \ldots, M\}: D_{Y_{m}}\left(2^{j}, k\right)=$ $\left\langle 2^{-j / 2} \psi_{0}\left(2^{-j} t-k\right) \mid Y_{m}(t)\right\rangle[20,21]$. Their sample covariances, or wavelet spectra, are given by the $M \times M$ symmetric positive semidefinite random matrices

$$
S\left(2^{j}\right) \triangleq \frac{1}{n_{j}} \sum_{k=1}^{n_{j}} D\left(2^{j}, k\right) D\left(2^{j}, k\right)^{*}, \quad n_{j}=2^{-j} N,
$$

with $N$ the sample size and $j$ the octave. Now let $\left\{\lambda_{1}\left(2^{j}\right), \ldots\right.$, $\left.\lambda_{M}\left(2^{j}\right)\right\}$ be the eigenvalues of $S\left(2^{j}\right)$, and let

$$
\vartheta_{m}\left(2^{j}\right) \triangleq \log _{2}\left(\lambda_{m}\left(2^{j}\right)\right)
$$

be the corresponding log-eigenvalues. Over analysis scales $2^{j_{1}}, \ldots, 2^{j_{2}}$, the wavelet estimator $\left(\hat{H}_{1}, \ldots, \hat{H}_{M}\right)$ for $\left(H_{1}, \ldots, H_{M}\right)$ is defined by means of weighted linear regressions of $\vartheta_{m}\left(2^{j}\right)$ as

$$
\hat{H}_{m}=\left(\sum_{j=j_{1}}^{j_{2}} w_{j} \vartheta_{m}\left(2^{j}\right)\right) / 2-\frac{1}{2}, \quad \forall m=1, \ldots, M,
$$

where $w_{j}$ are linear regression weights $[12,14]$. It can be shown that $\left(\hat{H}_{1}, \ldots, \hat{H}_{M}\right) \stackrel{P}{\rightarrow}\left(H_{1}, \ldots, H_{M}\right)$ under mild assumptions.

\section{BOOTSTRAP-BASED BIAS-REDUCED ESTIMATION}

\subsection{Repulsion effect of wavelet log-eigenvalues $\vartheta_{m}\left(2^{j}\right)$}

The study of the repulsion effect of eigenvalues of Hermitian matrices has a long history $[22,23]$ and it appears in the random matrix literature for a number of canonical models [16]. In addition, even in the case of sample covariance matrices formed from i.i.d. entries, the so-named level spacings between ordered eigenvalues are often nonnegligible in the nonclassical setting where the dimension $M$ of the system is comparable to the sample size $N$ (cf. [24-27]). For random wavelet covariance matrices, the separation effect is supposed to be naturally more pronounced for eigenvalues driven by equal Hurst exponents, for which they tend to differ only by a constant factor. For this reason, we focus on this case.

A study of wavelet log-eigenvalue repulsion is depicted in Fig. 1 (left) for $M=2$ (top) and $M=12$ (bottom) and $H_{m}=H=$ $0.6, m=1, \ldots, M$; for greater readability, the theoretical slopes $j\left(2 H_{m}+1\right)$ have been subtracted from $\vartheta_{m}\left(2^{j}\right)$. Details on the numerical simulation are given in Section 4 below. In light of the proven consistency of wavelet log-eigenvalues for Hurst exponents $[12,14]$, the naive expectation would be to observe nearly parallel straight wavelet log-eigenvalue lines as the octave $j$ increases. However, what the plots show is rather different. In general, for a fixed $M$, wavelet log-eigenvalue lines tend to divert away from each other as the octave $j$ increases. In turn, the average vertical distance between $\vartheta_{1}$ and $\vartheta_{M}$ also grows as a function of $M$. For any $M$, unreported studies further reveal that wavelet log-eigenvalues drift apart as the sample size $N$ or the effective sample size $n_{j}=N / 2^{j}$ decreases. This provides a computational demonstration of a repulsion effect of wavelet log-eigenvalues as a function of the parameters $M$, $N$ and $j$. In Fig. 1 (right column), wavelet log-eigenvalues are depicted for $\rho_{m, m^{\prime}}=0.5, m \neq m^{\prime}$, showing that the repulsion effect is also present, but weaker, when the components ofBm are correlated or have non-equal energy. In most practical situations where both $N$ and $M$ are fixed, this further implies that repulsion is more conspicuous at coarser scales. This increasing positive (for large $m$ ) or negative (for small $m$ ) bias of $\vartheta_{m}\left(2^{j}\right)$ as a function of $j$ introduces, in turn, bias in the estimation of Hurst exponents $H_{m}$.

\subsection{Multivariate wavelet bootstrap-based bias reduction}

Due to the general unavailability of closed-form expressions for wavelet log-eigenvalues, we resort to a bootstrap approach to obtain estimates of the bias of $\hat{\theta} \in\left\{\vartheta_{m}\left(2^{j}\right), \hat{H}_{m}\right\}, m=1, \ldots, M$. The main idea is to replace the ensemble equation $\mathbb{E}[\hat{\theta}]-\theta$ defining the bias for $\hat{\theta}$ with a sample version $\hat{\mathbb{E}}\left[\hat{\theta}^{*}\right]-\hat{\theta}$ that can be computed from a single realization of data, where $\hat{\mathbb{E}}\left[\hat{\theta}^{*}\right]$ denotes the average over a large number of bootstrap replicas $\hat{\theta}^{*}$ of $\hat{\theta}$ [28]. To obtain bootstrap replicas of $\vartheta_{m}\left(2^{j}\right)$ and $\hat{H}_{m}$, we construct a wavelet-domain bootstrap procedure that preserves the joint covariance structure of the wavelet coefficients. This is achieved by resampling the vector coefficients $D\left(2^{j}, k\right), k=1, \ldots, n_{j}$ in a (circular) block-bootstrap procedure, instead of resampling the wavelet coefficients of each component independently, which would destroy their covariance structure [29]. More precisely, $R$ block bootstrap resamples $D_{j}^{*(r)}=$ $\left(D^{*(r)}\left(2^{j}, 1\right), \ldots, D^{*(r)}\left(2^{j}, n_{j}\right)\right), r=1, \ldots, R$, are drawn by sampling with replacement $\left\lceil\operatorname{card}(Y) / L_{B}\right\rceil$ overlapping blocks of size $L_{B},\left(D\left(2^{j}, k\right), \ldots, D\left(2^{j}, k+L_{B}-1\right)\right), k=1, \ldots, n_{j}$, from the periodically extended samples $\left(D\left(2^{j}, 1\right), \ldots, D\left(2^{j}, n_{j}\right)\right)$. This procedure is repeated for each scale $j$ of interest, and bootstrap 

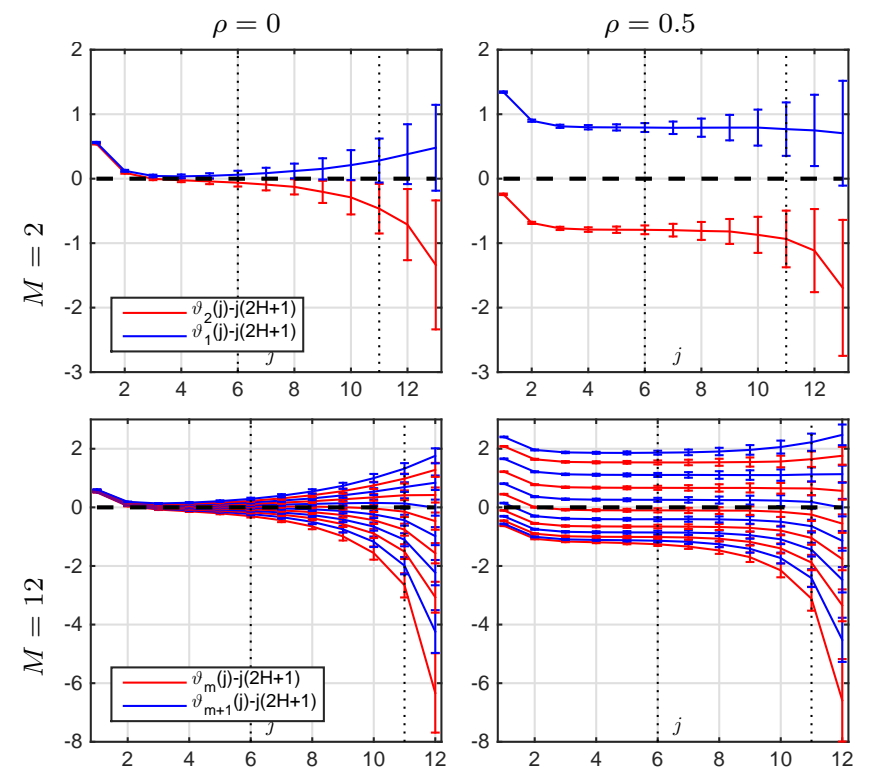

Fig. 1. Log-eigenvalues vs. scale. Averages and std of logeigenvalues $\vartheta_{m}(j)$ for 1000 independent realizations as a function of $j$, with ground truth $j\left(2 H_{m}+1\right)$ subtracted: $M=2, H=0.6$ (top row), $M=12, H=0.6$ (bottom row) with $\rho_{m, m^{\prime}}=0$ (left column) and $\rho_{m, m^{\prime}}=0.5$ (right column).

estimates $S^{*(r)}(j), \vartheta_{m}^{*(r)}\left(2^{j}\right)$ and $\hat{H}_{m}^{*(r)}$ are computed for each resample $D_{j}^{*(r)}$ as in (3-4). Finally, subtracting the bootstrap-based estimates for the bias, $\hat{\mathbb{E}}\left[\vartheta_{m}^{*}\left(2^{j}\right)\right]-\vartheta_{m}\left(2^{j}\right)$ and $\hat{\mathbb{E}}\left[H_{m}^{*}\right]-H_{m}$, from the estimates $\vartheta_{m}\left(2^{j}\right)$ and $\hat{H}_{m}$ yields the bootstrap bias corrected estimates [28]

$$
\begin{aligned}
\vartheta_{m}^{(b c)}\left(2^{j}\right) & \triangleq 2 \vartheta_{m}\left(2^{j}\right)-\frac{1}{R} \sum_{r=1}^{R} \vartheta_{m}^{*(r)}\left(2^{j}\right) \\
\hat{H}_{m}^{(b c)} & \triangleq 2 \hat{H}_{m}-\frac{1}{R} \sum_{r=1}^{R} \hat{H}_{m}^{*(r)} .
\end{aligned}
$$

\section{BIAS REDUCTION PERFORMANCE ASSESSMENT}

Simulation setup. To illustrate the eigenvalue repulsion effect in the wavelet domain and to assess the performance of the proposed bias reduction procedure, we resort to numerical simulation using $N_{M C}=1000$ independent realizations of $M$-variate ofBm of length $n=2^{16}$, with $\Sigma=\operatorname{Toeplitz}\left(1, \rho, \rho^{2}, \ldots, \rho^{(M-1)}\right)$. The mixing matrices $W$ are orthogonal random matrices generated independently for each realization. We study the cases $M \in\{2,12\}$ for $H_{m}=H=0.6$ and $\sigma_{m}=1, \forall m$, with correlation levels $\rho \in\{0,0.25,0.5,0.75\}$. Complementary results were obtained for instances where $H_{m} \neq H_{m^{\prime}}$; they lead to qualitatively similar conclusions and are not reported here for space reasons. We use Daubechies2 wavelets and scales $\left(j_{1}, j_{2}\right)=(6,11)$ for the Hurst exponent estimation, and $R=500$ bootstrap replica with block size $L_{B}=4$ ( $\approx$ length of the wavelet) for the bias reduction procedure.

Repulsion effect and bias reduction. Corresponding to the bottom left panel in Fig. 1, Fig. 2 (top row) provides a more detailed depiction of the (log) eigenvalue $\vartheta_{m}\left(2^{j}\right)$ repulsion effect for the instance $\rho=0$ and $M=12$. The figure shows the histograms
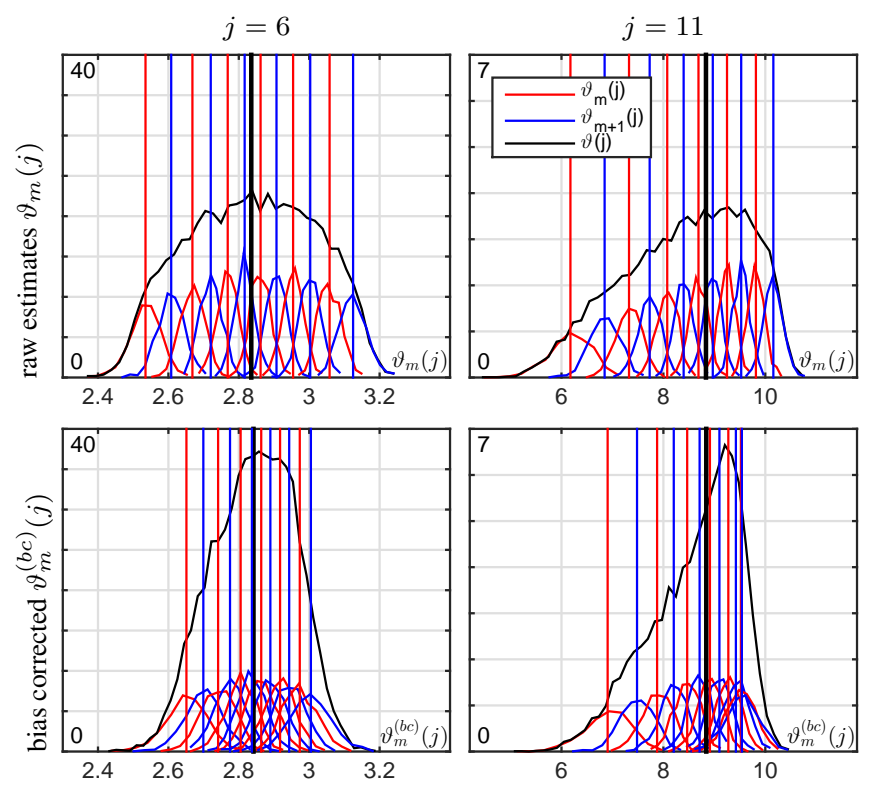

Fig. 2. Distribution of log-eigenvalues. Histograms of $\vartheta_{m}(j)$, $m=1, \ldots, 12$ for $\rho=0(j=6$, left column and $j=11$, right column), without (top row) and with (bottom row) bias correction; vertical bars indicate the average estimates $(\rho=0, H=0.6)$.

(over 1000 realizations) of $\vartheta_{m}\left(2^{j}\right)$, for each component $m$ separately (blue and red solid lines) together with average values for $\vartheta_{m}\left(2^{j}\right)$ (vertical bars), for scales $j=6$ and $j=11$ (left and right column, respectively). Also plotted are the overall histogram and average obtained by pooling the log-eigenvalues for all components (black solid lines and vertical bars). The repulsion effect of ordered wavelet log-eigenvalues $\vartheta_{m}(j), m=1, \ldots, M$, is clearly demonstrated: the individual histograms are spread out, resulting in spaced apart modes. Without repulsion, these modes would collapse onto one single value. A comparison of the results for $j=6$ and $j=11$ shows that these gaps get wider as we move from fine to coarse scales. For example, the distance between the averages of $\vartheta_{1}(j)$ and $\vartheta_{M}(j)$ increases from $\approx 0.6$ at scale $j=6$ to $\approx 4$ at scale $j=11$. This is also reflected in an enlarged support for the overall histogram at coarse scale.

Fig. 2 (bottom row) depicts the equivalent plots for bias corrected log-eigenvalues $\vartheta_{m}^{(b c)}(j)$. It shows that, for the bootstrapbased estimates, the gap between the smallest and the largest logeigenvalue is reduced by about $40 \%$ to $\approx 0.35$ at scale $j=6$ and $\approx 2.5$ at scale $j=11$, without modifying the overall average (indicated by vertical black bars). This demonstrates that the bootstrap bias reduction procedure is effective in counteracting the repulsion effect for wavelet log-eigenvalues, both at coarse and fine scales.

Fig. 2 also indicates that the supports of the sample distributions of each $\vartheta_{m}^{(b c)}(j)$ are enlarged with respect to their homologues $\vartheta_{m}\left(2^{j}\right)$. Therefore, the reduction in bias comes at the price of a slight increase in the variance.

Estimation of Hurst exponents $\underline{\boldsymbol{H}}$. Fig. 3 plots histograms of the estimated exponents $\hat{H}_{m}$ (left column) and of the bootstrapbased estimates $\hat{H}_{m}^{(b c)}$ (right column) for $\rho=\{0,0.25,0.5,0.75\}$ (top to bottom row, respectively) and $M=12$. It demonstrates that the scale-dependent strength of repulsion of the log-eigenvalues $\vartheta_{m}\left(2^{j}\right)$ discussed in the previous paragraph can result in signifi- 

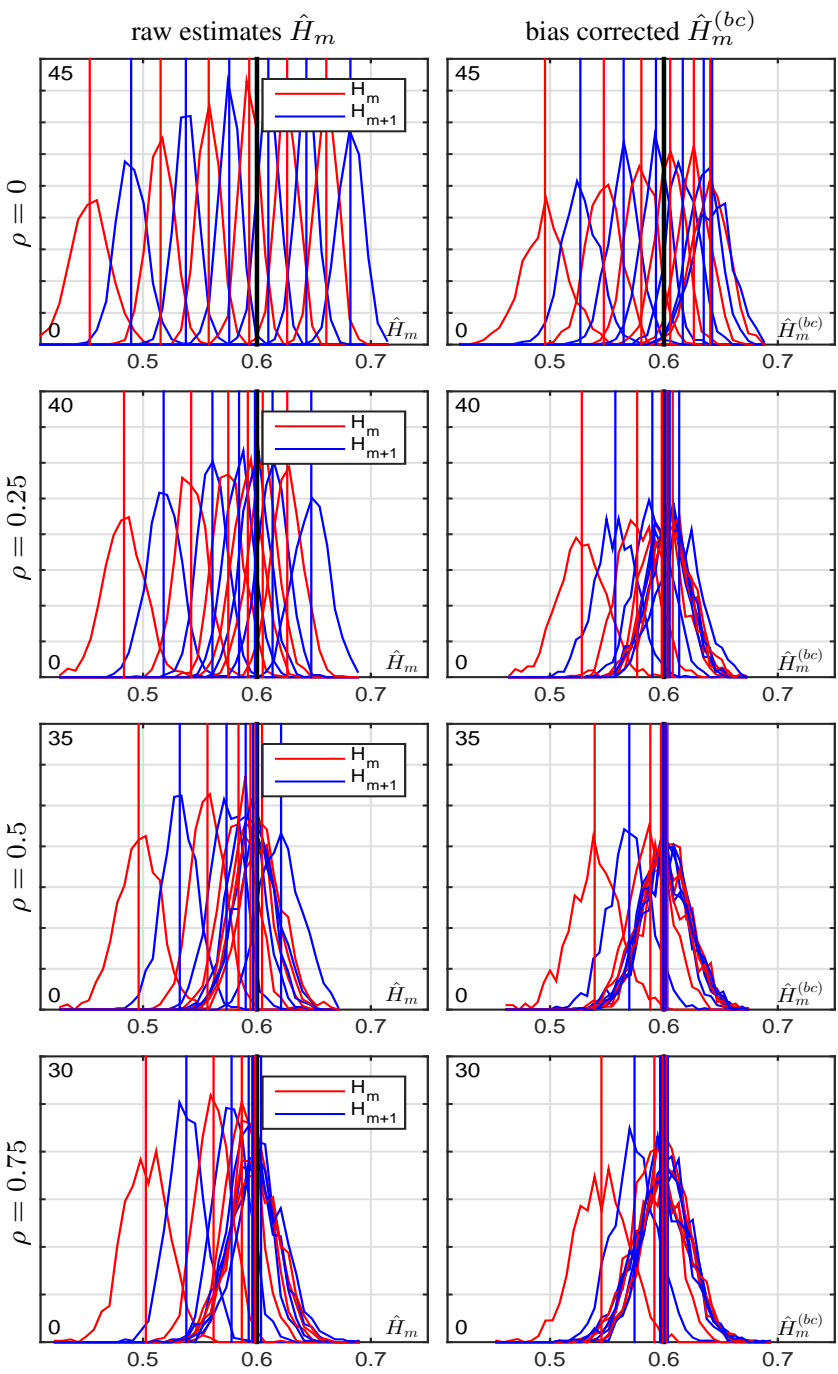

Fig. 3. Distribution of Hurst exponents. Histograms of estimates $\hat{H}_{m}, m=1, \ldots, 12$, without (left column) and with (right column) bias correction, for $\rho=\{0,0.25,0.5,0.75\}$ (top to bottom row, respectively); vertical bars indicate the average estimates $(H=0.6)$.

cant bias for $\hat{H}_{m}$. For example, for $\rho=0$, the average estimates range from $\hat{H}_{1}=0.45$ to $\hat{H}_{M}=0.68$, when theoretically $H_{m}=$ $H=0.6$. When $\rho$ increases, the bias becomes less dramatic but remains present; interestingly, it is the smallest (in terms of $\vartheta_{m}\left(2^{j}\right)$ and hence $\hat{H}_{m}$ ) components that are more affected by the repulsion effect. A comparison with Fig. 3 (right column) shows that the proposed bootstrap-based bias reduction procedure is effective and leads, e.g., for $\rho=0$, to average estimates ranging from $\hat{H}_{1}^{(b c)}=$ 0.5 to $\hat{H}_{M}^{(b c)}=0.64$. The procedure also provides good results for larger values of $\rho$ and yields essentially bias-free estimates for $\approx 7$ $(\rho=0.25), 9(\rho=0.5)$ and $10(\rho=0.75)$ out of the 12 components, as compared to $\approx 1,6$ and 7 out of 12 components for $\hat{H}_{m}$, respectively. This reduction in bias comes at the price of slightly broadened distributions, hence increased variability, for each individual $\hat{H}_{m}^{(b c)}$ as compared to $\hat{H}_{m}$.

Performance analysis. The above results show that the estimates

\begin{tabular}{|l|r|r|||r|r|c|}
\hline \multicolumn{7}{|c|}{$\boldsymbol{M}=\mathbf{2}, \boldsymbol{H}_{\boldsymbol{m}}=\mathbf{0 . 6}$} \\
\hline av. bias & $\hat{H}_{m}$ & $\hat{H}_{m}^{(b c)}$ & av. rmse & $\hat{H}_{m}$ & $\hat{H}_{m}^{(b c)}$ \\
\hline$\rho=0.00$ & 0.022 & $\mathbf{0 . 0 1 3}$ & $\rho=0.00$ & 0.030 & $\mathbf{0 . 0 2 6}$ \\
\hline$\rho=0.25$ & 0.006 & $\mathbf{0 . 0 0 0}$ & $\rho=0.25$ & $\mathbf{0 . 0 2 4}$ & $\mathbf{0 . 0 2 4}$ \\
\hline$\rho=0.50$ & 0.004 & $\mathbf{0 . 0 0 0}$ & $\rho=0.50$ & $\mathbf{0 . 0 2 4}$ & $\mathbf{0 . 0 2 4}$ \\
\hline$\rho=0.75$ & 0.004 & $\mathbf{0 . 0 0 1}$ & $\rho=0.75$ & 0.024 & $\mathbf{0 . 0 2 3}$ \\
\hline \hline \multicolumn{7}{|c|}{$\boldsymbol{M = 1 2 ,} \boldsymbol{H}_{\boldsymbol{m}}=\mathbf{0 . 6}$} \\
\hline av. bias & $\hat{H}_{m}$ & $\hat{H}_{m}^{(b c)}$ & av. rmse & $\hat{H}_{m}$ & $\hat{H}_{m}^{(b c)}$ \\
\hline$\rho=0.00$ & 0.058 & $\mathbf{0 . 0 3 0}$ & $\rho=0.00$ & 0.060 & $\mathbf{0 . 0 3 5}$ \\
\hline$\rho=0.25$ & 0.037 & $\mathbf{0 . 0 1 0}$ & $\rho=0.25$ & 0.041 & $\mathbf{0 . 0 2 2}$ \\
\hline$\rho=0.50$ & 0.025 & $\mathbf{0 . 0 0 5}$ & $\rho=0.50$ & 0.034 & $\mathbf{0 . 0 2 3}$ \\
\hline$\rho=0.75$ & 0.022 & $\mathbf{0 . 0 0 4}$ & $\rho=0.75$ & 0.033 & $\mathbf{0 . 0 2 3}$ \\
\hline
\end{tabular}

Table 1. Average (over components $m$ and independent Monte Carlo realizations) absolute bias and rmse values (left and right, respectively) for $\hat{H}_{m}$ and $\hat{H}_{m}^{(b c)}$, for several values for $\rho$ and for $M=2$ (top) and $M=12$ (bottom, best results appear boldfaced).

$\hat{H}_{m}^{(b c)}$ have smaller bias than $\hat{H}_{m}$ but also indicate larger variance. To quantify the potential overall benefit of the proposed bootstrapbased bias reduced estimates for $\underline{H}$, we evaluate the performance in terms of the average (over components $m$ and independent Monte Carlo realizations) absolute bias and root mean squared error (rmse), for several levels of correlation $\rho$ as well as $M=2$ and $M=12$ components. The results are summarized in Table 1 and lead to the following conclusions. The proposed estimator $\hat{H}_{m}^{(b c)}$ yields systematic performance improvements over the standard estimator $\hat{H}_{m}$, both in terms of bias and in terms of rmse. For the scenarios considered here, bias is reduced by up to an order of magnitude, and rmse values by up to a factor 2 . The improvements in bias and rmse values are less visible for $M=2$ than for $M=12$ because the strength of the repulsion effect for eigenvalues, and thus bias, grows with $M$. For the same reason, the improvement in performance is more significant for smaller values for $\rho$. Overall, these results unambiguously demonstrate the effectiveness and systematic practical benefits of the proposed bias reduced estimation for multivariate self-similarity.

\section{CONCLUSIONS}

In this work, we study the repulsion effect of eigenvalues of wavelet covariance matrices and its impact on the wavelet eigenanalysisbased estimation of multivariate Hurst exponents. We construct and assess an original wavelet domain bootstrap-based bias correction procedure assuming a single finite-length multivariate time series is available. Extensive numerical simulations show that the proposed method significantly improves the finite sample performance of the wavelet eigenvalue regression, with up to a tenfold (twofold) reduction in bias (rmse). The work opens up several new methodological perspectives, including the study of the benefits of doublebootstrapping for higher-order bias correction, and the construction of statistical tests for the equality of self-similarity exponents in multivariate data. Future applied work includes the modeling of neuroscientific multivariate data (fMRI, E/MEG). 


\section{REFERENCES}

[1] G. Samorodnitsky and M. Taqqu, Stable non-Gaussian random processes, Chapman and Hall, New York, 1994.

[2] B. B. Mandelbrot and J. W. van Ness, "Fractional Brownian motion, fractional noises and applications," SIAM Reviews, vol. 10, pp. 422-437, 1968.

[3] V. Pipiras and M. S. Taqqu, Long-Range Dependence and SelfSimilarity, vol. 45, Cambridge University Press, 2017.

[4] J. Beran, Statistics for Long-Memory Processes, Chapman \& Hall/CRC, Boca Raton, 1994.

[5] J. Beran, Y. Feng, S. Ghosh, and R. Kulik, Long-Memory Processes: Probabilistic Properties and Statistical Methods, Springer-Verlag, New York, 2013.

[6] P. Flandrin, "Wavelet analysis and synthesis of fractional Brownian motion," IEEE Trans. Info. Theory, vol. 38, no. 2, pp. 910-917, 1992.

[7] D. Veitch and P. Abry, "A wavelet-based joint estimator of the parameters of long-range dependence," IEEE Trans. Info. Theory, vol. 45, no. 3, pp. 878-897, 1999.

[8] M. Maejima and J. D. Mason, "Operator-self-similar stable processes," Stochastic Processes and their Applications, vol. 54, no. 1, pp. 139-163, 1994.

[9] J. D. Mason and Y. Xiao, "Sample path properties of operatorself-similar Gaussian random fields," Theory of Probability \& Its Applications, vol. 46, no. 1, pp. 58-78, 2002.

[10] G. Didier and V. Pipiras, "Integral representations and properties of operator fractional Brownian motions," Bernoulli, vol. 17, no. 1, pp. 1-33, 2011.

[11] G. Didier and V. Pipiras, "Exponents, symmetry groups and classification of operator fractional Brownian motions," Journal of Theoretical Probability, vol. 25, pp. 353-395, 2012.

[12] P. Abry and G. Didier, "Wavelet eigenvalue regression for $n$ variate operator fractional Brownian motion," Journal of Multivariate Analysis, vol. 168, pp. 75-104, November 2018.

[13] P. Abry, G. Didier, and H. Li, “Two-step wavelet-based estimation for Gaussian mixed fractional processes," To appear in Statistical Inference for Stochastic Processes, pp. 1-61, 2018.

[14] P. Abry and G. Didier, "Wavelet estimation for operator fractional Brownian motion," Bernoulli, vol. 24, no. 2, pp. 895928, 2018.

[15] G. W. Anderson, A. Guionnet, and O. Zeitouni, An Introduction to Random Matrices, vol. 118 of Cambridge Studies in Advanced Mathematics, Cambridge University Press, Cambridge, 2010.

[16] T. Tao, Topics in Random Matrix Theory, vol. 132, American Mathematical Society, 2012.

[17] J. Yao, S. Zheng, and Z.D. Bai, Sample Covariance Matrices and High-Dimensional Data Analysis, Cambridge University Press, 2015.

[18] P.-O. Amblard and J.-F. Coeurjolly, "Identification of the multivariate fractional Brownian motion," IEEE Trans. Signal Proces., vol. 59, no. 11, pp. 5152-5168, 2011.

[19] H. Wendt, G. Didier, S. Combrexelle, and P. Abry, "Multivariate Hadamard self-similarity: testing fractal connectivity," Physica D, vol. 356-357, pp. 1-36, 2017.
[20] I. Daubechies, Ten Lectures on Wavelets, vol. 61, SIAM, 1992.

[21] S. Mallat, A Wavelet Tour of Signal Processing, Academic Press, San Diego, CA, 1998.

[22] J. von Neumann and E.P. Wigner, "Über das Verhalten von Eigenwerten bei adiabatischen Prozessen," in The Collected Works of Eugene Paul Wigner, pp. 294-297. Springer, 1993.

[23] P. Lax, Linear Algebra, John Wiley \& Sons Inc., 1996.

[24] V. Plerou, P. Gopikrishnan, B. Rosenow, L. A. N. Amaral, T. Guhr, and H. E. Stanley, "Random matrix approach to cross correlations in financial data," Physical Review E, vol. 65, no. 6, pp. 066126, 2002.

[25] G. Akemann, J. Fischmann, and P. Vivo, "Universal correlations and power-law tails in financial covariance matrices," Physica A: Statistical Mechanics and its Applications, vol. 389, no. 13, pp. 2566-2579, 2010.

[26] Z. Bai and J. W. Silverstein, Spectral Analysis of Large Dimensional Random Matrices, vol. 20, Springer, New York, $2^{\text {nd }}$ edition, 2010.

[27] D. Taylor, J. G. Restrepo, and F. G. Meyer, "Ensemble-based estimates of eigenvector error for empirical covariance matrices," arXiv preprint arXiv:1612.08804, 2018.

[28] A. M. Zoubir and D. R. Iskander, Bootstrap Techniques for Signal Processing, Cambridge University Press, 2004.

[29] S. N. Lahiri, Resampling Methods for Dependent Data, Springer, New York, 2003. 